\title{
Recent Progress on Optical Regeneration of Wavelength-Division-Multiplexed Data
}

Patki, Pallavi G.; Guan, Pengyu; Li, Lu; Lakoba, Taras I.; Oxenløwe, Leif Katsuo; Vasilyev, Michael; Galili, Michael

Published in:

IEEE Journal of Selected Topics in Quantum Electronics

Link to article, DOI:

10.1109/JSTQE.2020.3025482

Publication date:

2021

Document Version

Peer reviewed version

Link back to DTU Orbit

Citation (APA):

Patki, P. G., Guan, P., Li, L., Lakoba, T. I., Oxenløwe, L. K., Vasilyev, M., \& Galili, M. (2021). Recent Progress on Optical Regeneration of Wavelength-Division-Multiplexed Data. IEEE Journal of Selected Topics in Quantum Electronics, 27(2), [7700812 ]. https://doi.org/10.1109/JSTQE.2020.3025482

\section{General rights}

Copyright and moral rights for the publications made accessible in the public portal are retained by the authors and/or other copyright owners and it is a condition of accessing publications that users recognise and abide by the legal requirements associated with these rights.

- Users may download and print one copy of any publication from the public portal for the purpose of private study or research.

- You may not further distribute the material or use it for any profit-making activity or commercial gain

- You may freely distribute the URL identifying the publication in the public portal 


\title{
Recent Progress on Optical Regeneration of Wavelength-Division-Multiplexed Data
}

\author{
Pallavi G. Patki, Pengyu Guan, Member, IEEE, Lu Li, Taras I. Lakoba, Leif Oxenløwe, \\ Member, IEEE, Michael Vasilyev, Senior Member, IEEE, and Michael Galili, Member, \\ IEEE
}

(Invited Paper)

\begin{abstract}
Practical applications of all-optical regeneration require from it a graceful scalability with the number of wavelength-division-multiplexed (WDM) channels. We describe several main approaches to WDM-compatible regeneration and review in greater detail two recent demonstrations of 16-channel regeneration: one using time-lensing and the other using groupdelay-management. We discuss scalability, practical issues, and outlook for these WDM regeneration methods.
\end{abstract}

Index Terms-nonlinear-optical signal processing, optical regeneration, nonlinear fiber optics, time lensing, dispersion management.

\section{INTRODUCTION}

$\mathrm{O}$ PTICAL regeneration of data signals has been a topic of research for several decades. The prospect of having an optically transparent way of improving signal quality rather than using high-speed electrical discriminators has intrigued researchers resulting in numerous interesting propositions on how to solve this challenge.

In optical fiber transmission, noise from optical amplifiers and the presence of fiber nonlinearities will gradually degrade the modulation of optical data signals, eventually to the point of preventing correct detection. One of the main attractions of optical fiber communication is the very large bandwidth of optical fibers and optical amplifiers, which allows many highspeed data signals to propagate in the link simultaneously using wavelength-division multiplexing (WDM). This possibility of sharing the fiber infrastructure and associated optical amplifiers between many optical data signals is undoubtedly a key reason for the success of optical fiber technology for communication. It is unlikely that an optical regeneration scheme, which does not support WDM, will be attractive in most systems. The main challenge to an optical regeneration scheme when applying it to WDM signals it to avoid strong inter-channel crosstalk in the structure intended to regenerate the signals. Many of the regeneration schemes that work well for single-channel systems

Manuscript received July X, 2020; revised YYYY Y, 2020; accepted ZZZZ Z, 2020. DTU work was supported by the Research Center SPOC (DNRF123). UT Arlington work was supported in part by the National Science Foundation grants DMS-0507429 / 0507540 and ECCS-0925706 / 0925860.

Pengyu Guan, Leif Oxenløwe, and Michael Galili are with DTU Fotonik, Technical University of Denmark, DK-2800 Kgs. Lyngby, Denmark (e-mails: pengu@ fotonik.dtu.dk, lkox@ fotonik.dtu.dk,mgal@fotonik.dtu.dk).

Pallavi G. Patki was with the Department of Electrical Engineering, University of Texas at Arlington, Arlington, TX, 76019, USA. She is now with Infinera Corp., Bangalore 560025, India (e-mail: patkipallavi@ gmail.com). rely on the nonlinear response of either optical fibers or semiconductor devices. In both cases, this nonlinear response will inherently modulate any co-propagating signals causing strong crosstalk unless care is taken to prevent this from happening. Three main approaches have been followed to avoid this detrimental effect. They will be outlined in the following section together with a discussion of their respective benefits and drawbacks.

An area of research closely related to optical regeneration is that of optical nonlinearity compensation. The objective here is to utilize the fact that optical nonlinearities, even though they often appear as noise to a data signal, are in fact deterministic effects that are well described and understood. Consequently, several schemes have been developed and pursued to compensate for signal distortions caused by optical nonlinearities in fiber transmission. The most common approaches rely on either optical phase conjugation (OPC) [1] or digital back propagation (DBP) [2]. A general limitation of optical nonlinearity compensation is that it only addresses deterministic signal distortions. It does not allow correcting signal distortions caused by stochastic noise. The presence of stochastic noise, however, can reduce the ability to compensate for optical nonlinearities [3].

Optical regeneration, which is the topic of this paper, is a fundamentally different approach where one does not attempt to undo specific effects that may have degraded the signal but rather attempts to re-shape, re-time and re-amplify the signal (3R regeneration). Often, only a subset of these regeneration steps is considered, and in this paper, we mainly discuss reshaping of the signal ( $2 \mathrm{R}$ regeneration). In practice, this requires a nonlinear transfer function between input and output signal along the dimension of data modulation, typically phase or amplitude. The targeted result when a noisy data signal is subjected to such a nonlinear transfer function is compression of the noise distributions around the designated modulation

Lu Li was with the Department of Electrical Engineering, University of Texas at Arlington, Arlington, TX, 76019, USA. He is now with SubCom, Eatontown, NJ 07724, USA (e-mail: luli@ subcom.com).

Michael Vasilyev is with the Department of Electrical Engineering, University of Texas at Arlington, Arlington, TX, 76019, USA (e-mail: vasilyev@uta.edu).

Taras I. Lakoba is with the Department of Mathematics and Statistics, University of Vermont, Burlington, VT 05401, USA (e-mail: Taras.Lakoba@uvm.edu). 
levels. This process does not eliminate any bit errors that have occurred due to noise but it does make the signal more robust to further noise accumulation. Fig. 1 illustrates the principle of regeneration using a nonlinear transfer function. The distributions indicated by the dashed blue curves represent twolevel data modulation affected by noise resulting in data symbol realizations following a normal distribution centered on the target modulation levels. A moderate amount of noise (blue dashed line) is present in the input signal, allowing the nonlinear transfer function of the regenerator to compress these
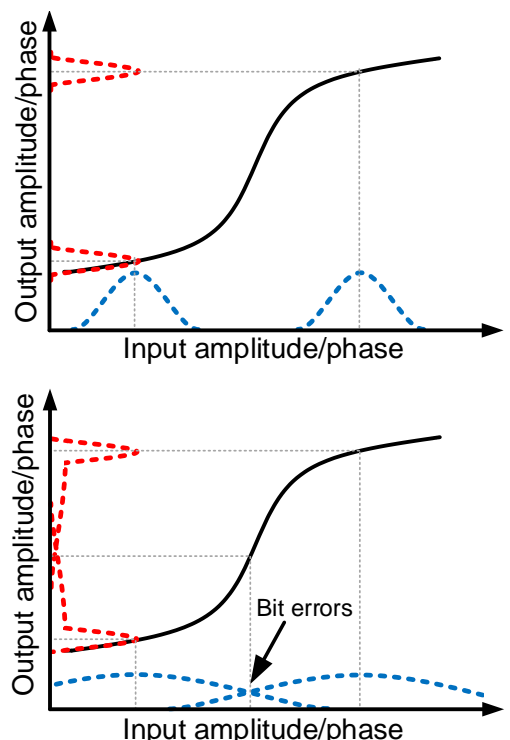

Fig. 1. Principle of regeneration by reshaping noise distributions using a nonlinear transfer function between input and output signals. Top compression of distributions for well separated input modulation levels. Bottom - redistribution of noise for significantly overlapping noise distributions at input.

distributions of data symbol realizations as indicated by the red dashed line. This compression of the distributions allows further noise to be added to the data signal without significant overlap of the distributions to occur. As the occurrence of bit errors depends on the overlap of these distributions, the regeneration allows further noise accumulation, e.g., due to further transmission, without bit errors occurring at the same rate as in the case without regeneration. Fig. 1 bottom illustrates the scenario where the input signal has been affected by noise causing significant overlap of the distributions of data symbol realizations. This overlap causes bit errors when the signal is received, and the regenerator will not be able to improve bit errors caused by this overlap. Even an ideal step-like regenerator transfer function would force data symbols in this overlap region to the wrong level in the same way as the thresholding mechanism would do in a receiver. Regeneration in this scenario may, however, still improve the signal's robustness to further transmission and reduce the rate of further error accumulation.

In Section II we will outline the main approaches which have been followed in reported WDM regeneration demonstrations. An excellent review [4] gave a detailed account of related research prior to year 2012. Hence in this paper, we will concentrate on later work. In Sections III and IV we will review and discuss the two recent demonstrations of WDM regeneration which have currently achieved the best results one based on time lenses to convert WDM into time-division multiplexing (TDM) and the other based on careful group delay management inside the regenerator. Section V will conclude with the outlook for practical deployment of optical regeneration.

\section{ApProaches to WDM REgeneration}

The reported WDM regeneration demonstrations generally fall into one of three classes of WDM regenerators. This classification will not apply to all reported demonstrations but it does apply to the vast majority.

The first approach relies on demultiplexing the data channels and processing them in separate single-channel optical regenerators [5-9]. This approach allows the direct application of regenerators, which work well for single channels, in WDM systems. As each regenerator unit in this approach only processes a single wavelength channel, crosstalk inside the regenerator is not a concern. The approach is sketched in Fig. $\underline{2}$, where the WDM channels are demultiplexed, typically, in an arrayed waveguide grating (AWG) and then individually regenerated before finally being combined in a multiplexer. This approach has primarily been attractive for demonstrations based on integrated optical structures where semiconductor devices are used for regeneration [5, 6]. The small size of the individual components allows the whole structure to be kept quite compact, even though this approach mandates a large number of components, if many WDM channels are to be regenerated. So far, structures for regenerating up to eight WDM channels have been reported [6]. With the continuous development of photonic integration, it is quite possible that structures allowing a larger number of channels could be realized. A drawback of this scheme is that the component count and power consumption scale proportionally to the number of WDM channels.

Some regenerator components can be reused by several channels by employing additional degrees of freedom, e.g., polarization [10] and direction of propagation [11, 12], to avoid the crosstalk. Regeneration of up to four WDM channels in a single polarization-maintaining highly-nonlinear fiber (HNLF) has been demonstrated by using polarization multiplexing and bidirectional propagation [13].

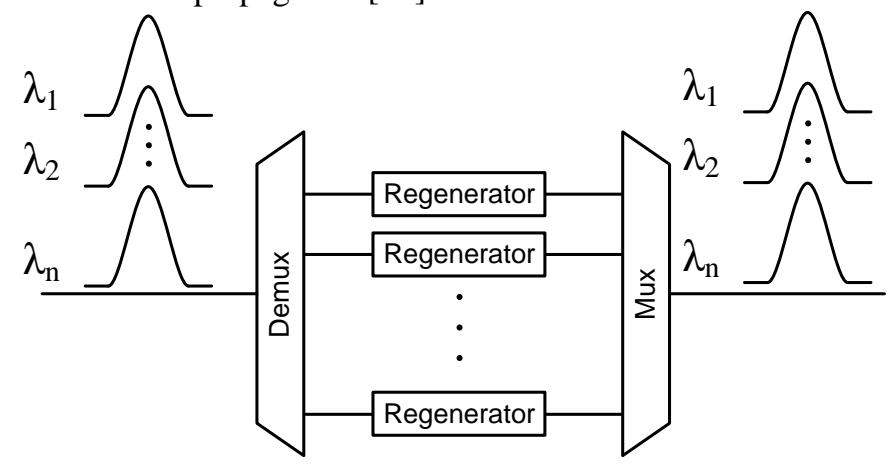

Fig. 2. Illustration of WDM regeneration based on single-channel regenerators.

The second approach to reducing inter-channel crosstalk in an optical regenerator is to use time interleaving of data channels [14-17]. This approach is particularly attractive when using regenerators based on nonlinear devices with a very fast 
nonlinear response. In practice, most of the demonstrations following this approach have been based on optical fiber as a nonlinear medium. Here the ultrafast electronic nonlinearity, the Kerr effect, has a response time of only a few femtoseconds [18], which allows data pulses to be processed in very close succession without incurring any crosstalk due to memory in the regenerator. This approach is less easily compatible with conventional WDM systems, since each WDM channel must contain data pulses with a low duty cycle, and strict time synchronization is required between the WDM channels. The operating principle of this WDM regeneration scheme is sketched in Fig. 3.

As long as the time between data pulses exceeds the relaxation time of the optical nonlinearity, this scheme provides good isolation between the processed channels. Various schemes have been devised to relax the requirements to the WDM system from this regenerator while still benefitting from the very low crosstalk. In Section III we will discuss in more detail how a time-lens-based system relaxes the requirement of having short pulses in the WDM channels. Using that approach 16 WDM channels have been simultaneously regenerated [19].

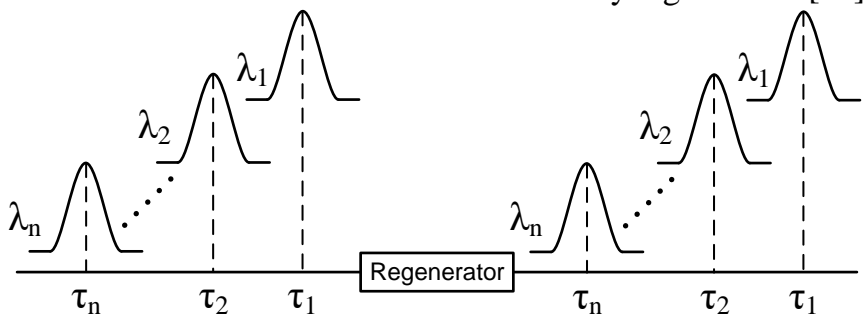

Fig. 3. The pulses belonging to different WDM channels are separated in time, avoiding co-propagation through the regenerator, which could otherwise have caused crosstalk.

The third approach utilizes the dispersion of the nonlinear medium to reduce the interaction among the WDM channels. Indeed, in the presence of sufficiently high dispersion, the significant difference in group velocities of different WDM channels makes their data trains rapidly walk off from one another. The parasitic cross-phase modulation (XPM) among the channels, therefore, is averaged over long data patterns and results only in creation of a benign constant nonlinear phase shift for each channel, instead of a detrimental patterndependent XPM crosstalk. Parasitic four-wave mixing (FWM) is also drastically suppressed by dispersion. This method is typically used in the regenerators of on-off-keyed (OOK) return-to-zero (RZ) signals, based on self-phase modulation (SPM), such as Mamyshev regenerator discussed in Section IV. The key dilemma of this approach is to ensure fast group walkoff between neighboring WDM channels, but at the same time prevent rapid spreading of the data pulses, which is also caused by dispersion. Quick disintegration of pulses can prevent accumulation of SPM that is necessary for regeneration. Conventional dispersion management employing combinations of normal- and anomalous-dispersion fibers has helped [4, 20, 21], but even in that case the dispersion dilemma is resolved only by spacing WDM channels far from one another in frequency domain, resulting in low spectral efficiency. This has limited regenerators with conventional dispersion management to under four WDM channels [4, 20, 21].

The dispersion dilemma inherent to the third approach can be resolved, however, by employing non-traditional dispersion management relying on elements with discontinuous dispersion spectra. This method has recently resulted in regeneration of 16 WDM channels [22] and will be discussed in Section IV, including some previously unpublished setup optimization data in Section IVA.

\section{WDM REGENERATION BASED ON OPTICAL TIME LENSES}

As discussed above, a key challenge to optical regeneration is the crosstalk, which occurs between WDM channels inside the regenerator. In one of the two recent demonstrations to reach 16-channel WDM regeneration, this limitation was overcome by converting 16 WDM channels into a single TDM signal using an optical time lens. This signal was then regenerated and finally converted back to WDM channels by a second time lens. Similar to regular lenses, the so-called time lens structure has the possibility to perform a Fourier-transform-like process between the features of a waveform in the time domain and the spectral domain [23, 24]. This transformation can be performed with a scaling determined by the specific implementation of the system. The basic principle of this optical Fourier transformation (OFT) is illustrated in Fig. Fig. 4 for the case of converting WDM channels into well-separated TDM channels.

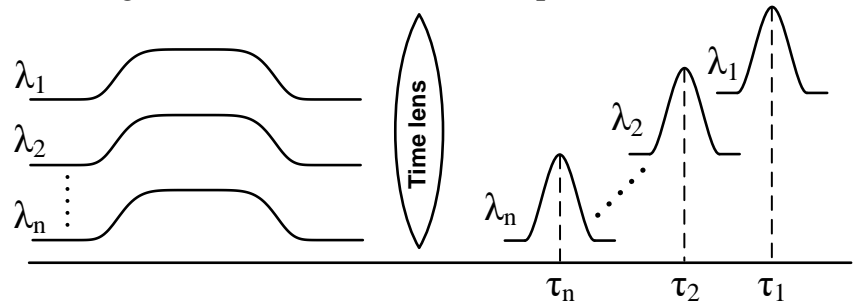

Fig. 4. A time lens system is used to perform optical Fourier transformation of WDM channels into temporally separated TDM channels.

Conversion between WDM and TDM channels has been demonstrated for various applications [25, 26] and is particularly useful in the context of optical regeneration, as it allows the use of regeneration schemes which are not inherently compatible with WDM. Furthermore, the conversion between WDM and TDM is transparent to the data modulation in both phase and amplitude. This makes it an attractive approach for advanced modulation formats, which combine modulation of both phase and amplitude. Phase-sensitive amplifiers (PSAs) have attracted a lot of attention for optical regeneration because of the step-like transfer function that can be achieved for signal

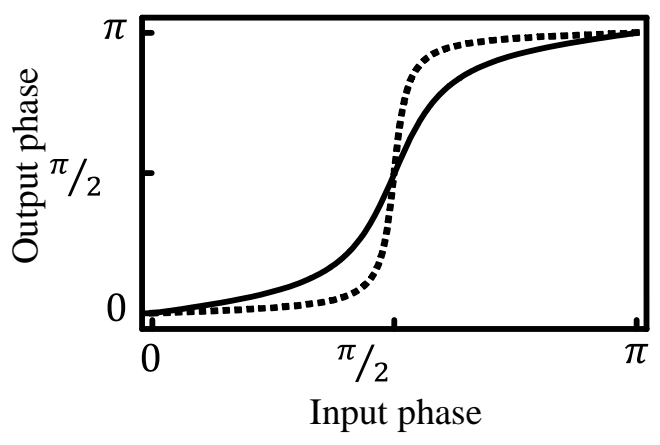

Fig. 5. Phase transfer function of PSA-based regenerator. The dashed and solid lines are for PSA gains of 4 and 2, respectively. 


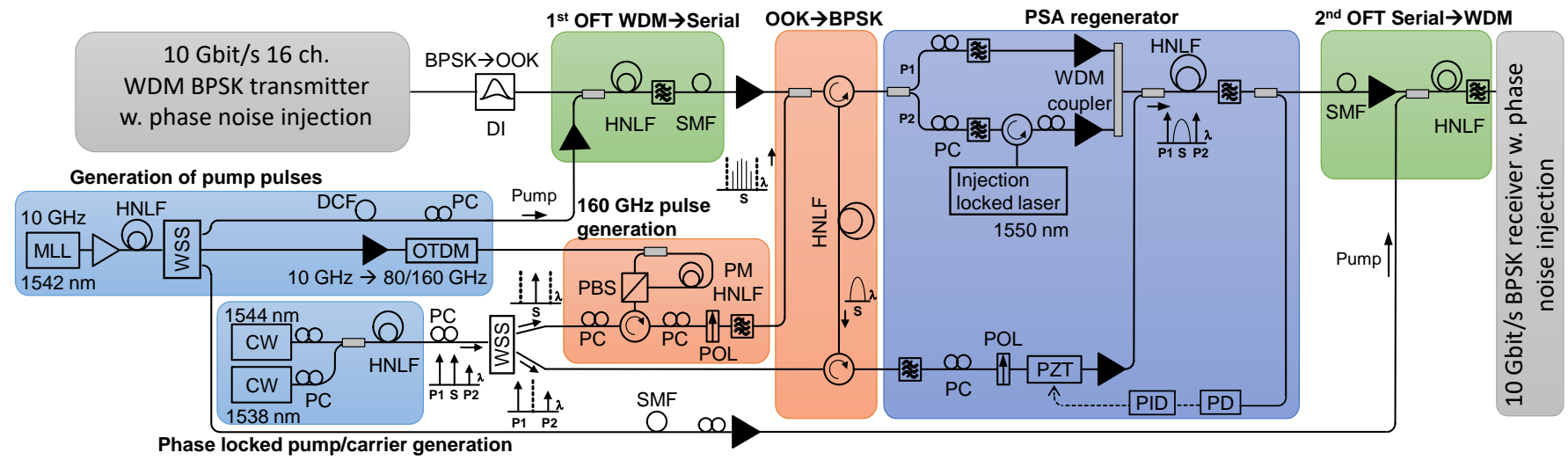

Fig. 6. Experimental setup for time-lens-based WDM regenerator.

phase $[27,28]$. For the demonstration of time-lens-based WDM regeneration, a PSA-based regenerator was used in order to obtain a sufficiently strong discrimination between the logical levels of the data modulation. Figure 5 shows phase transfer functions of the PSA-based regenerator scheme for different gains in the PSA. The dashed and solid lines are for PSA gains of 4 and 2, respectively.

The regeneration scheme in the 16-channel demonstration is the same as the one studied extensively in [29] for singlechannel operation. The steep transfer function, which can be achieved in a PSA with moderate gain, is clearly attractive for regeneration. In order to combine PSA regeneration with the WDM-to-TDM conversion provided by the time lens system, it is necessary to consider the inter-pulse coherence in the generated TDM signal. The performance of the PSA relies on having a stable phase relation between all the interacting fields - the pumps and the signal. In the general case where no coherence is assumed between separate WDM channels, the TDM signal generated by the time lens will not have a stable phase relation between consecutive pulses. In the 16-channel regeneration demonstration this is overcome by demodulating the binary phase-shift-keying (BPSK) modulated WDM channels in a single delay interferometer. The resulting amplitude shift keying (ASK) modulated pulses subsequently use XPM to modulate the phase of a train of pulses carved from a continuous wave (CW) laser. In this way, the binary information stored in the phase of the individual WDM channels is transferred to the phase of a train of pulses carved from a single laser. This ensures that all the data tributaries are aligned in phase, allowing for subsequent phase-sensitive regeneration of them all. Following the regeneration of the data, the TDM signal is converted back to separate WDM channels in a second time lens. In the demonstration, the regenerated WDM channels were placed on the same frequency grid as the input channels. With different configuration of the second time lens, the frequency grid of the regenerated channels can be chosen to be different from the input. The setup used to demonstrate this regeneration scheme is illustrated in Fig. 6. 16 10-Gbit/s BPSK modulated WDM channels are generated in the transmitter and launched into the regenerator. To characterize the regenerator performance, transmitter and receiver are equipped with phase modulators to inject controlled amounts of phase noise into the signal. The pump pulses for the two OFT- stages are derived from a $10 \mathrm{GHz}$ mode-locked laser (MLL). Pump pulses with the correct properties in terms of spectrum and chirp are generated by spectral broadening in highly nonlinear fiber (HNLF) and subsequent application of filtering and chromatic dispersion. After the first OFT, the XPM-stage and regenerator are configured to ensure stable phase between pumps and signal in the PSA. Phase-locked pumps are generated by FWM between the light from two free-running lasers $(1544 \mathrm{~nm}$ and $1538 \mathrm{~nm})$. In this way, the light from the 1538-nm laser and the generated idler at $1550 \mathrm{~nm}$ can be used as PSA pumps, while the light from the 1544-nm laser is used as the carrier for the re-modulated BPSK signal. This, in combination with an optical phase-locked-loop to track and compensate ambient effects in the fiber setup, ensures stable operation of the PSA.

The demonstration of the regenerator confirmed strong regeneration of signals primarily degraded by phase noise. To test the performance of the regenerator, the 16 WDM channels were exposed to random modulation of their phase both before and after passing through the regenerator. This was done to emulate in-line operation of the regenerator, where the aim of regeneration is to improve the robustness of the signal to further noise addition. An improvement in bit error rate (BER) of approximately one order of magnitude was found for the 16 WDM channels as seen in Fig. 7. With no noise injected into the signal at the transmitter and the receiver, the regenerator clearly degrades the performance of the signal. This is not

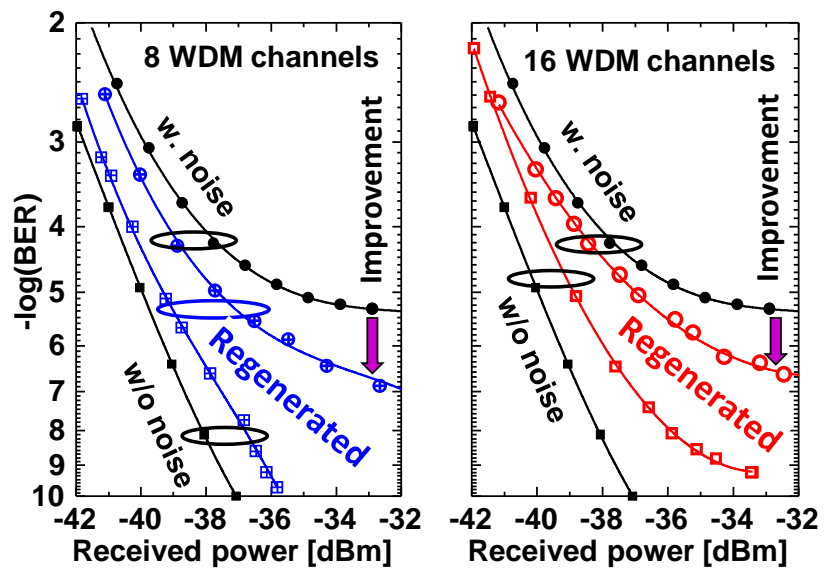

Fig. 7. BER results for 8- and 16-channel regeneration. The black lines with filled symbols show the performance without regeneration. The purple arrow indicates the improvement of the signal with noise by regeneration. 
surprising, as the regeneration system has several stages that add some amount of noise to the signal. If the initial signal has very little noise, the performance will be dominated by the noise of the regenerator. However, if the signal is subjected to noise before and after the regenerator, the nonlinear transfer function
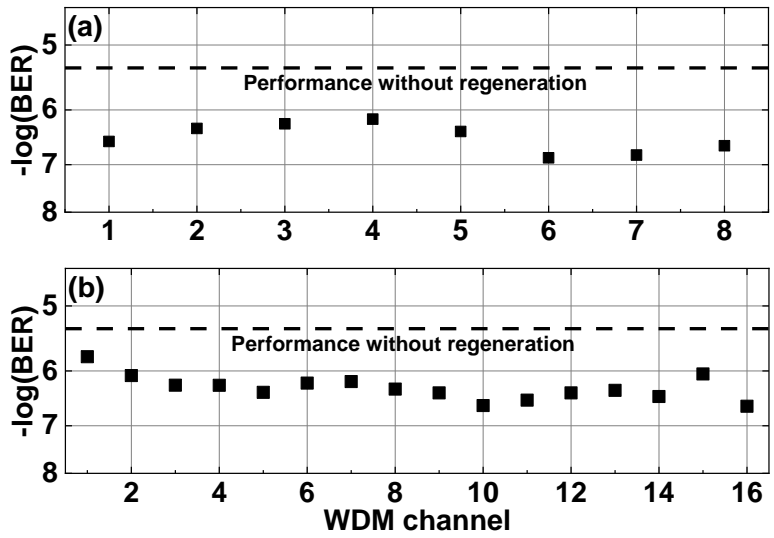

Fig. 8. Performance of all WDM channels with and without regeneration. (a) is the 8-channel case and (b) is the 16-channel case. The dashed line indicates the BER of the WDM channels in the presence of phase noise, but without regeneration. The markers indicate performance after regeneration.

will compress the noisy levels of the input signal, making it more robust to further noise. This behavior is clearly seen from the BER curves where the purple arrow indicates the improvement in achievable BER, which is approximately one order of magnitude for both 8- and 16-channel regeneration. This performance increase is found for all WDM channels as seen in Fig. 8, where the BER with regeneration is shown by the square markers while the BER for the same amount of phase noise but without regeneration is shown by the dashed line. Due to slight imperfections in the preparation of the OFT pump pulses, some variation in performance is seen between the channels after regeneration. The original WDM signal has the same performance for all channels. The detailed results and analysis for this system are found in [19].

This scheme for WDM regeneration is promising, since it allows the use of optical regeneration schemes that are not inherently compatible with WDM. Furthermore, the scheme allows changing the wavelengths and grid of the WDM channels. The main challenge still faced by this scheme is the requirement for synchronization of the WDM channels. The time lens converting the WDM signal into TDM requires the data pulses belonging to the different WDM channels to arrive at the time lens at the same time. The main consequence of this is that all data channels belonging to a WDM signal will need to be generated according to the same master clock. Delay management can then align their arrival at the time lens. As long as these requirements are met, the time-lens-based WDM regeneration is a promising scheme for regenerating phasemodulated data channels. Currently, it seems promising to extend the scheme to quaternary phase-shift keying (QPSK) modulation, because de-modulation, re-modulation and phasesensitive regeneration have all been demonstrated to work for QPSK in a very similar scheme in [30].

\section{WDM REGENERATION BASED ON GROUP-DELAY MANAGEMENT}

Before introducing the dispersion-management method that enables highly scalable WDM regeneration, let us first discuss the operating principle of the regenerator based on SPMinduced spectral broadening followed by frequency-offset spectral filtering, a.k.a. Mamyshev regenerator [31]. It was originally designed for a single WDM channel, which undergoes spectral broadening due to SPM in a nonlinear fiber, as shown in Fig. 9 left. Under proper conditions, the width of the broadened spectrum becomes proportional to the peak power of the input signal pulse, transferring the fluctuations of pulse magnitude into the fluctuations of the spectral width while keeping the power spectral density nearly constant. When a portion of the broadened spectrum is selected by an optical bandpass filter (OBPF) with width similar to the width of the input spectrum, this produces an output pulse whose magnitude no longer depends on the input power fluctuations, resulting in regeneration of "ONE" symbols. Moreover, if the filter is offset
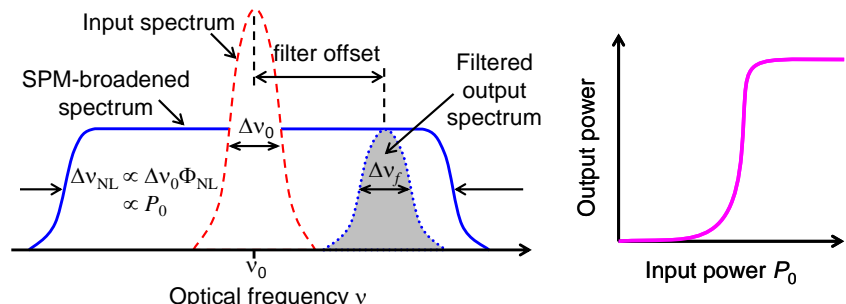

Fig. 9. (Left) Single-channel Mamyshev 2R regenerator is based on signal's spectral broadening by self-phase modulation (SPM), followed by off-center bandpass filtering. (Right) Resulting input-output power transfer curve. (a) Group-delay-managed (GDM) nonlinear medium

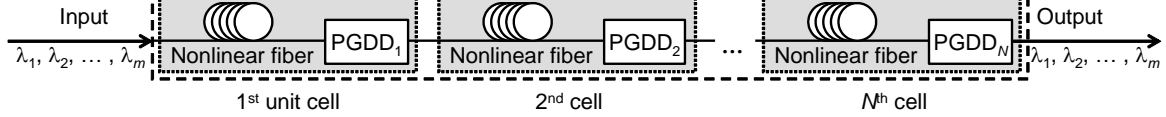

(b)

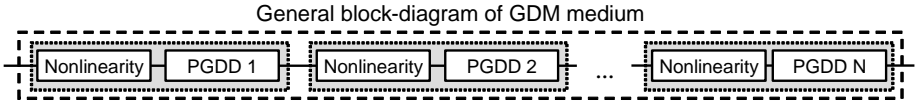

Potential planar-lightwave-circuit implementation $\left(\sim 1 \mathrm{~cm}^{2}\right)$

(c)

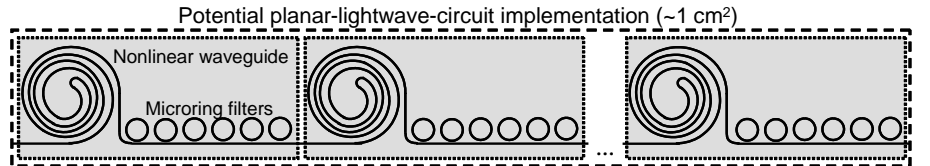

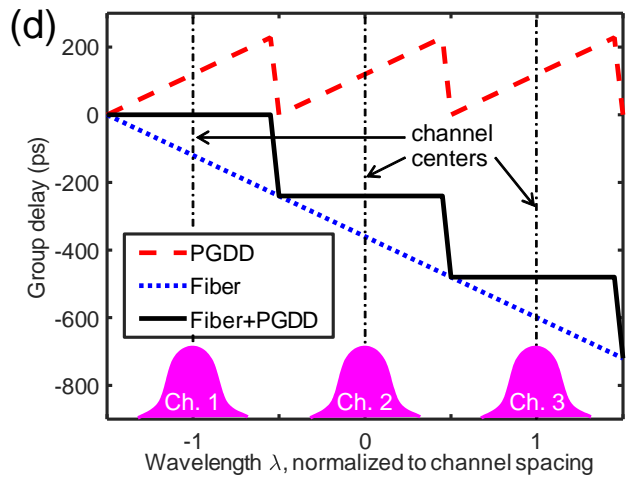

Fig. 10. Group-delay-managed nonlinear medium (a) in its implementation using nonlinear fiber and PGDDs, (b) in its general form, and (c) in its potential onchip implementation. (d) Group delay spectra of PGDD (dashed), DCF (dotted), and their combination comprising one GDM unit cell (solid). The selected spectral region covers 3 WDM channels. 
in frequency from the input signal, then any noise between the pulses is too weak to experience SPM and hence is not picked up by the OBPF, resulting in regeneration of "ZERO" symbols as well. The input-output power transfer curve of Mamyshev regenerator is close to an ideal step threshold function (Fig. 9 right).

While Mamyshev regenerator is extremely robust and works flawlessly with various pulse shapes, its use in WDM case is constrained by the dispersion dilemma described in Section II. To resolve this dilemma, i.e., to ensure fast interchannel walk-off without spreading of each channel's pulses, a novel nonlinear medium is needed, which must have a very large dispersion between the channels and nearly zero dispersion within the bandwidth of each channel. The medium with such a discontinuous dispersion does not occur naturally, but can be artificially synthesized, as shown in Fig. 10 a-c, from short pieces of conventional nonlinear dispersive medium separated by periodic group-delay devices (PGDDs), which are phase filters with sawtooth-like group-delay spectra (see dashed line in Fig. 10d). When PGDD is combined with highly dispersive nonlinear medium such as dispersion-compensating fiber (DCF, dotted line in Fig. 10d), the resulting group-delay spectrum of "fiber + PGDD" combination (we will refer to it as "unit cell") becomes staircase-like (solid line in Fig. 10d). Since dispersion $D$ is the derivative of the group-delay spectrum, then, by cascading many of these unit cells, one can build the desired effective nonlinear medium (referred to as group-delaymanaged, or GDM, medium) with nearly zero dispersion within each channel, but with very large dispersion between the neighboring WDM channels. If the fiber within the unit cell is short enough, then over the entire GDM medium the pulse width experiences only a slight periodic "breathing," leaving the pulse intact (see Fig. 11). Although currently commercially available PGDDs are built from either chirped fiber Bragg gratings [32] or from cascaded Gires-Tournois etalons [33, 34], the PGDDs can be also integrated with nonlinear waveguides $[35,36,37]$ on a chip in the form of cascaded microresonators [38] or AWGs [39, 40], as shown in Fig. 10c.

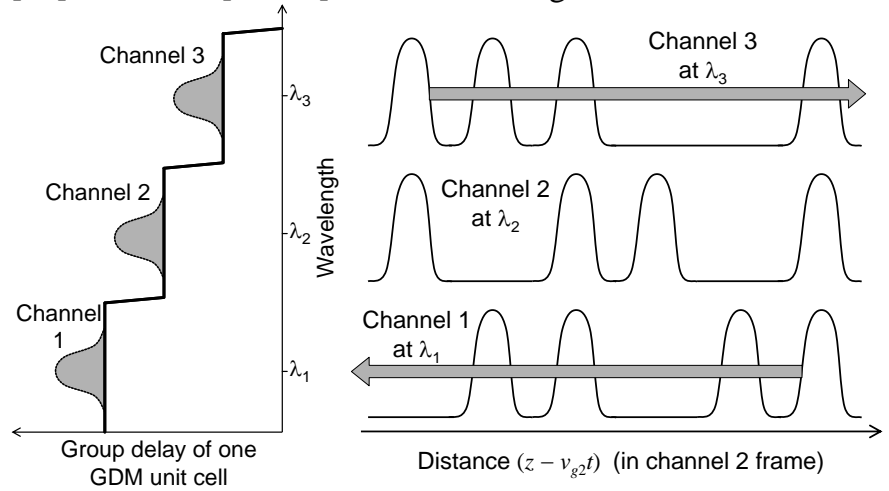

Fig. 11. Group-delay-managed (GDM) nonlinear medium ensures that bit sequences of the neighboring channels rapidly walk off from one another (large inter-channel dispersion), while the pulses within each channel remain intact (small intra-channel dispersion). Left graph shows group delay spectra of one GDM unit cell (thick solid black line), as well as the locations of the spectra of 3 neighboring channels (shaded areas with dotted borders). Right graph shows bit sequences of the same three channels versus propagation distance shown here in the frame of channel 2, which propagates through the GDM with group velocity $v_{g 2}$. Channel 3 propagates faster and channel 1 propagates slower than channel 2 .
Our initial GDM proposal [41] called for at least 16 unit cells, but a latter work [42] has found a very robust regime with slightly anomalous residual dispersion of each unit cell that only required 5-6 cells. Experimental demonstrations of multichannel $2 \mathrm{R}$ regeneration in this Section are carried out in the latter regime [42].

In all presented experiments of this Section the nonlinear fiber is the conventional dispersion-compensating fiber (DCF) with nonlinear constant $\gamma \approx 5(\mathrm{~W} \times \mathrm{km})^{-1}$, dispersion $D=-120$ $\mathrm{ps} / \mathrm{nm} / \mathrm{km}$ ), and attenuation $\approx 0.5 \mathrm{~dB} / \mathrm{km}$. All signals are OOK modulated by a $2^{31}-1$ pseudo-random bit sequence (PRBS) and carved into 50\% RZ pulses. To ensure the worst case of interchannel nonlinearities (XPM and FWM), all WDM signals are co-polarized. In order to properly characterize the $2 \mathrm{R}$ regeneration performance, we decorrelate the clock frequencies and the bit patterns between the neighboring channels, as explained in Sections IVA and IVB below. Without proper clock decorrelation, one might observe unrealistically optimistic regeneration performance (e.g., when the 50\% RZ pulses from the neighboring channels are interleaved in time), which cannot be achieved in a practical communications system, where the channels have independent (uncorrelated) clocks.

\section{A. Optimization of multi-channel $2 R$ regeneration in a recirculating-loop setup}

To verify the parameters of the theoretically-predicted multichannel regeneration regime [42], we need to be able to easily vary the number of "fiber + PGDD" unit cells in the regenerator. Hence, we have built a recirculating loop [43, 44], where we used only one "fiber + PGDD" unit cell and passed the signals through it multiple times (Fig. 12) to achieve the effect of concatenating multiple identical cells. In contrast to recirculating loops typically used in studying ultra-long-haul transmission, we have designed our loop to work with as little as a few hundred meters of fiber (few $\mu$ s round-trip time) by using fast (< $50 \mathrm{~ns}$ response time) loop switches AOS1, AOS2 and diagnostic equipment gates (switches AOS3, AOS4 in front of the optical spectrum analyzer (OSA) and digital communication analyzer (DCA), respectively, in Fig. 12).

The recirculating-loop setup uses a single "fiber + PGDD" unit cell with $1.25 \mathrm{~km}$ of DCF and $\approx+20 \mathrm{ps} / \mathrm{nm}$ unit cell residual dispersion [42]. Two sets of 400-GHz-spaced WDM channels are interleaved to form twelve 200-GHz-spaced channels $(1542.54 \ldots 1560.20 \mathrm{~nm})$ modulated by two independent 9.953$\mathrm{Gb} / \mathrm{s}$ pattern generators (PGs) with slightly different and uncorrelated clock frequencies. We also add a 2-bit delay between the adjacent channels within each set, decorrelating their bit patterns originating from the same PG by a $0.5-\mathrm{km}-$ long DCF. This ensures that we study a realistic worst-case scenario of co-polarized uncorrelated WDM channels, and average the performance over various initial delays between the pulses of neighboring channels. We degrade the channel selected for BER measurements by $\pm 25 \%$ amplitude jitter via adding a crosstalk from another laser tuned to the measured channel's wavelength. In the final optimized 12-channel experiment each channel's average power is $\sim 60 \mathrm{~mW}$ at the DCF input. The net loss and gain in the loop are carefully 


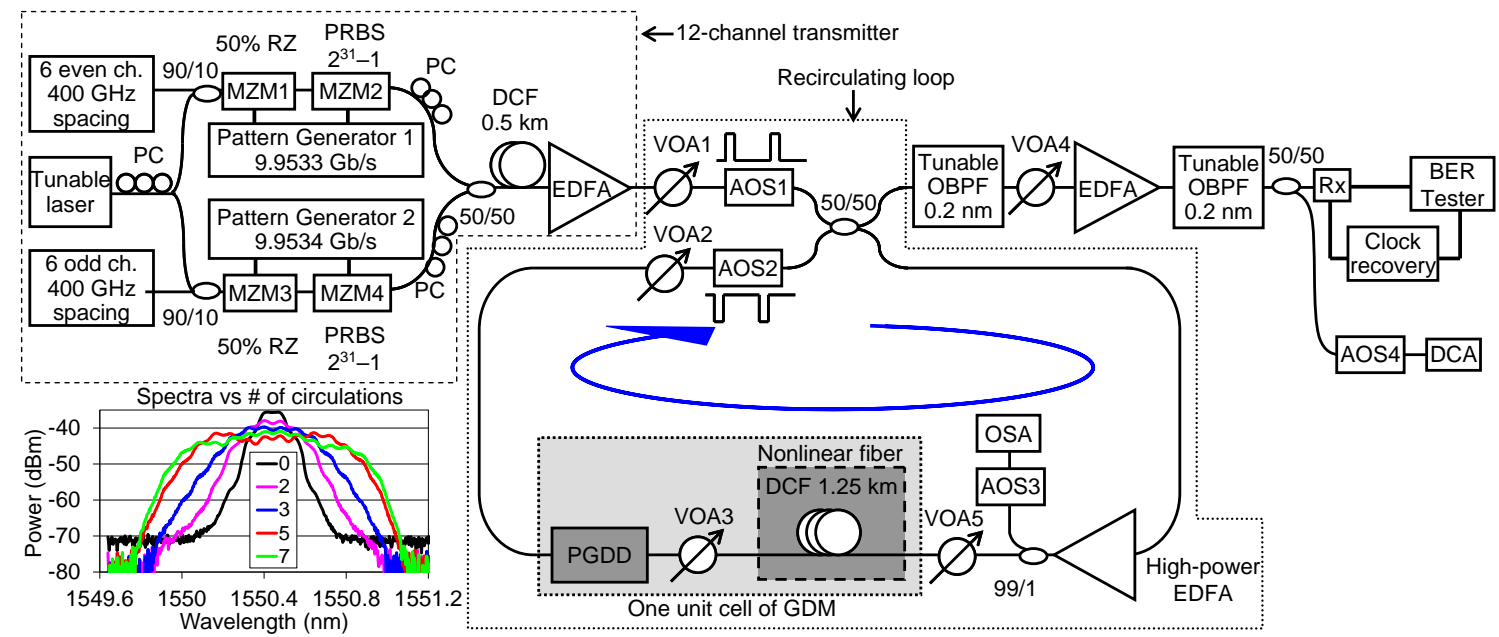

Fig. 12. Experimental recirculating-loop setup for investigation and optimization of multi-channel 2R regeneration. PC: polarization controller, MZM: electrooptic Mach-Zehnder modulator, EDFA: erbium-doped fiber amplifier, AOS: acousto-optic switch, VOA: variable optical attenuator, DCF: dispersioncompensating fiber, OBPF: optical bandpass filter, OSA: optical spectrum analyzer, DCA: digital communication analyzer. The inset shows SPM-broadened spectra of channel $6(1550.5 \mathrm{~nm})$ vs. number of circulations.

balanced. Pulsed gate coinciding with timing of a particular circulation in the loop controls the inputs to the OSA, BER tester, and DCA. After $N$ circulations, the regenerated signals are selected, one at a time, by a 0.2-nm-wide tunable OBPF, which is offset from the original signal's wavelength, and are subsequently detected by an optically pre-amplified receiver that includes another identical OBPF. The concatenated bandwidth of the two OBPFs is $\approx 18 \mathrm{GHz}$.

After each pass (circulation), the spectrum of each WDM channel is increasingly broadened by SPM, as shown in the inset of Fig. 12.
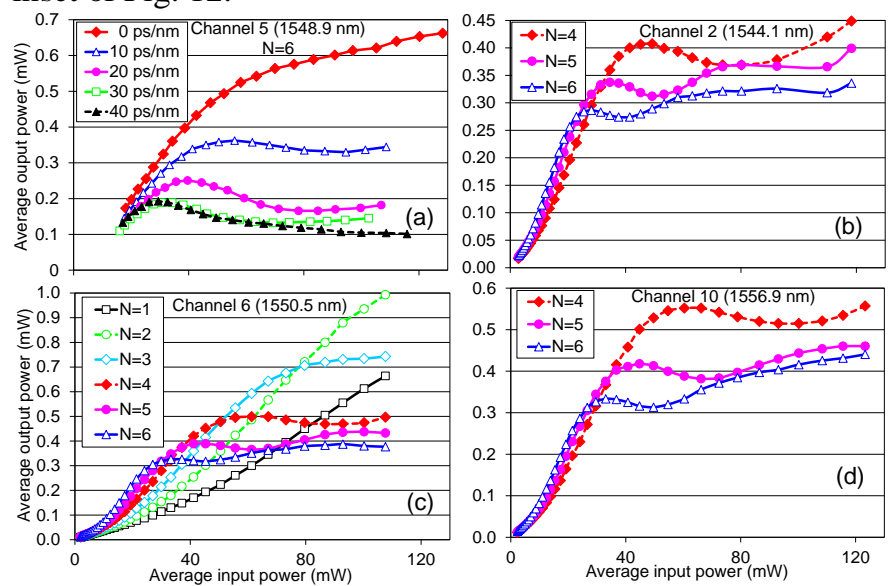

Fig. 13. Input-output power transfer curves: (a) for channel $5(1548.9 \mathrm{~nm})$ after $N=5$ circulations for various values of the residual dispersion of each unit cell, with OBPF detuning of $+0.16 \mathrm{~nm}$; for channels (b) 2 (1544.1 nm), (c) 6 (1550.5 $\mathrm{nm})$, and (d) $10(1556.9 \mathrm{~nm})$ for $10 \mathrm{ps} / \mathrm{nm}$ value of the residual dispersion of each GDM unit cell and OBPF detuning of $+0.15 \mathrm{~nm}$.

Figure 13a shows the input-output power transfer curves for channel $5(1548.5 \mathrm{~nm})$, obtained with residual dispersion per unit cell varying from 0 to $+40 \mathrm{ps} / \mathrm{nm}$ by tuning the PGDD. In these curves, the output power represents the average power of the output signal selected by the OBPFs with $+0.16 \mathrm{~nm}$ detuning, and the input power is the average power of the input to the first "fiber + PGDD" unit cell. After 6 circulations, the curve with $10 \mathrm{ps} / \mathrm{nm}$ residual dispersion shows the flattest plateau. After 5 circulations (not shown), $20 \mathrm{ps} / \mathrm{nm}$ residual dispersion results in the flattest curve.
Figures $13 \mathrm{~b}-\mathrm{d}$ show the input-output power transfer curves for three representative channels, obtained with residual dispersion per unit cell of $10 \mathrm{ps} / \mathrm{nm}$ and $+0.15 \mathrm{~nm}$ OBPF detuning. As can be seen from the curves for channel 6 (Fig. $13 \mathrm{c}$ ), the plateau does not appear until circulation number $N=4$, because only at this point the spectrum becomes sufficiently broadened for the signal to be selected by the detuned filter. While plateau exists for $N=4,5$, and 6 , we choose $N=5$, owing to its flatter shape, to clean the amplitude fluctuations of the input pulses. For $N=5$, optimum residual dispersion is +20 $\mathrm{ps} / \mathrm{nm}$.
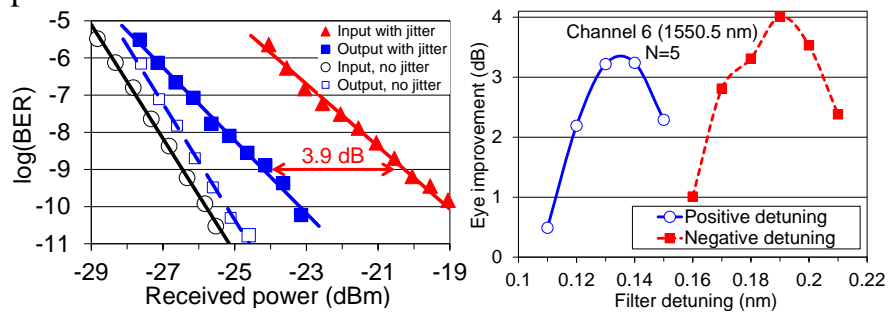

Fig. 14. (Left) BER curves for amplitude-jitter-degraded signals before (red triangles) and after the regeneration (blue squares) for channel $6(1550.5 \mathrm{~nm})$. BERs for signals without amplitude jitter are also shown before (black open circles) and after (blue open squares) the regeneration. $N=5$, OBPF detuning is $+0.15 \mathrm{~nm}$. (Right) Sensitivity of eye-opening improvement of channel 6 $(1550.5 \mathrm{~nm}$ ) after $N=5$ circulations to the amount of OBPF detuning.

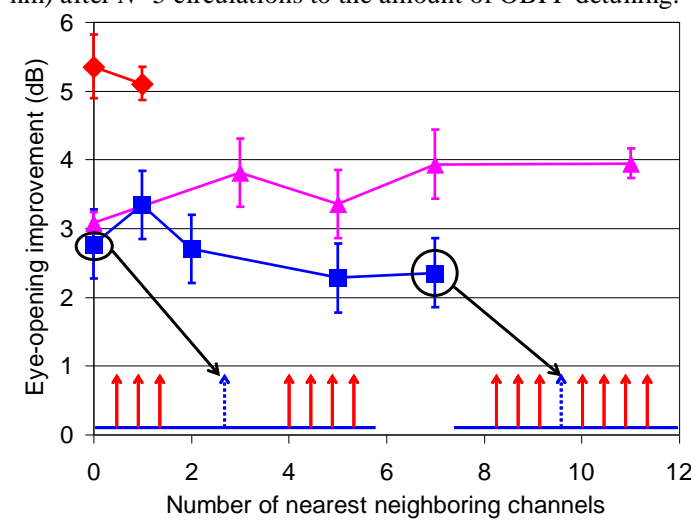

Fig. 15. Eye-opening improvement at $\mathrm{BER}=10^{-9}$ for the central $1550.5-\mathrm{nm}$ channel after $N=5$ circulations versus the number of other simultaneously regenerated channels. Different traces correspond to three separate experiments described in the text and should not be compared to one another. 
Using a 10-Gb/s signal after 5 circulations as the regenerated output, we have studied the regeneration performance with various numbers of channels. Although $2 \mathrm{R}$ regeneration cannot improve the intrinsic BER of the signal, the compression of noise of "ZERO" and "ONE" symbols makes the signal more robust with respect to subsequent noise addition. This improvement (a.k.a. "eye-opening improvement") is quantified by the reduction of the signal power level at the receiver's optical pre-amplifier entrance that is required to obtain BER = $10^{-9}$, as shown in Fig. 14 (left) for central channel 6 (1550.5 $\mathrm{nm}$ ) in a 12-channel experiment (the other channels are characterized similarly). Figure 14 (right) shows that the eyeopening improvement is robust with respect to moderate changes in OBPF detuning. The detuning tolerance is good enough to potentially permit the use of independent DFB or tunable lasers with an interleaver, which could perform OBPF function simultaneously on all channels. Although the merits of a regenerator are ultimately determined by the reach extension it offers to a specific real transmission system in the field, the eye-opening improvement is widely accepted as a laboratory measure of the regenerator's ability to suppress the signal's impairments and hence improve the system performance.

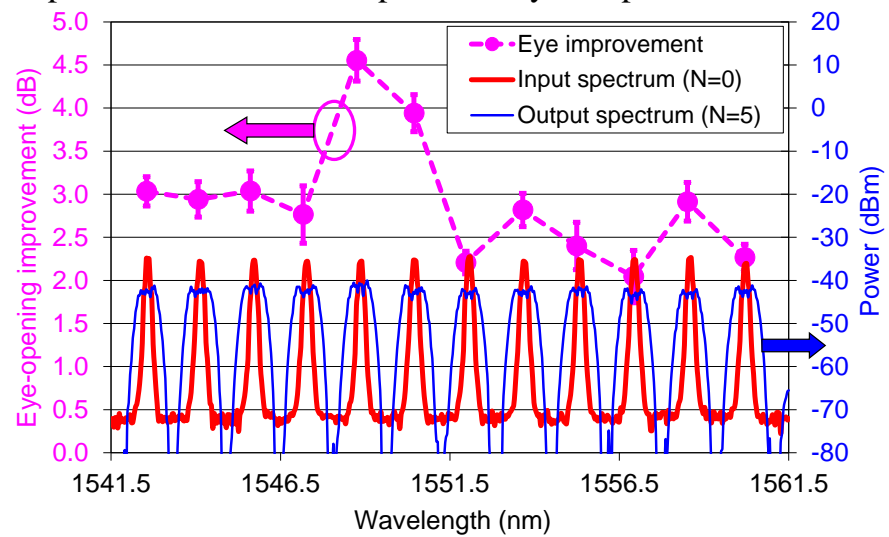

Fig. 16. Eye-opening improvements at $\mathrm{BER}=10^{-9}$ for 12 simultaneously regenerated channels (left axis; dashed line with filled circles); 12-channel spectra at the input (thin line) and output (thick line) of the regenerator (right axis), obtained with $0.1 \mathrm{~nm}$ resolution bandwidth. OBPF detuning is +0.15 $\mathrm{nm}$.

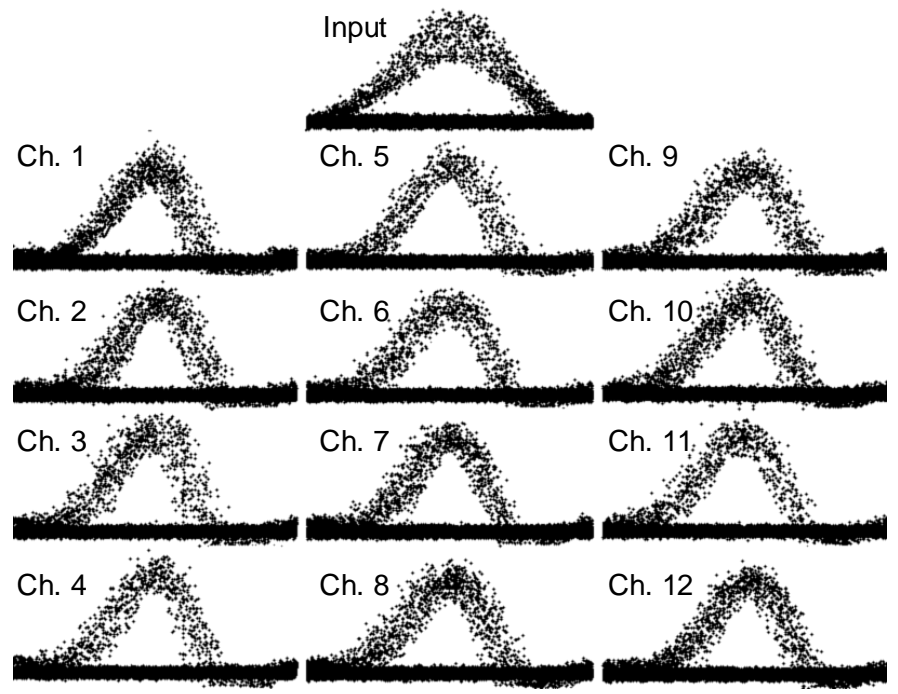

Fig. 17. Eye diagrams for signal input (after 0 circulations, no filter detuning) and output (after 5 circulations, $+0.15 \mathrm{~nm}$ filter detuning).
In three separate experiments summarized in Fig. 15, we have found that the eye-opening improvement of the central channel degraded by amplitude jitter does not show any noticeable degradation when the number of WDM channels on a 200-GHz-spaced grid is increased from 1 to 2 (diamonds in Fig. 15), from 1 to 12 (triangles), or when the number of the channel's closest neighbors on this grid is changed from 0 to 7 while keeping the total number of channels at 8 (squares). Our regenerator's performance is not degraded as we change the number of closest neighbor channels from 0 to 11 . Since only a few (5-7) closest neighbors have a potential to degrade the signal channel's performance, these results experimentally prove that there is no fundamental XPM or FWM limit on the number of channels regenerated in our scheme, i.e., adding more channels would not make regeneration worse.

The main results of the recirculating-loop experiment are presented in Figs. 16 and 17, demonstrating 2R regeneration of twelve 10-Gb/s channels with $200-\mathrm{GHz}$ spacing [22]. According to the spectra in Fig. 16, all channels undergo equal amount of SPM-based spectral broadening. The eye-opening data in Fig. 16, obtained from comparing the curves of BER versus receiver pre-amplifier's input power (examples of which were shown in Fig. 14 left), clearly indicate that the regenerator improves eye opening by more than $2 \mathrm{~dB}$ for all 12 channels. The eye diagrams in Fig. 17 also show significant reduction of the amplitude jitter after the regeneration.

\section{B. Demonstration of a stand-alone multi-channel $2 R$ regenerator}

With the insights gained from the recirculating-loop experiments, we have built a stand-alone $2 \mathrm{R}$ regenerator (Fig. 18a) consisting of 5 PGDDs and 6 sections of nonlinear fiber (Fig. 18b), as described below. We use 16 WDM channels $(1543.73 \ldots 1555.75 \mathrm{~nm})$ on a $100-\mathrm{GHz}$-spaced ITU grid, obtained by combining even and odd 8-channel sets. Since all signals are modulated by the same $10.7-\mathrm{Gb} / \mathrm{s}$ PRBS, we decorrelate the bit patterns between the even and odd WDM channel sets by 2 -km-long single-mode fiber (SMF) in the even-channel path. We also decorrelate the bit patterns within each of the even and odd channel sets by sending the combined signals through $2.5 \mathrm{~km}$ of DCF, producing a 5-bit pattern delay between the adjacent channels of each $200-\mathrm{GHz}$-spaced set. The intra-channel dispersion of this DCF is 67\%-compensated by a preceding PGDD (set to $+200 \mathrm{ps} / \mathrm{nm}$ ), while the dispersion accumulated between the channels is preserved. In addition, we decorrelate the clock frequencies of even and odd channels by frequency modulation (FM) of the clock of a single PG, in lieu of employing a second PG (see [22, 45] for more details). We emulate the real noise in transmission by modulating all channels with broadband (14-GHz-wide) Nyquist-Johnson noise generated by several cascaded electronic amplifiers. This noise separately modulates even and odd channels and is decorrelated among the channels by the same 2-km-long SMF and $2.5-\mathrm{km}$-long DCF combination used for data decorrelation. 


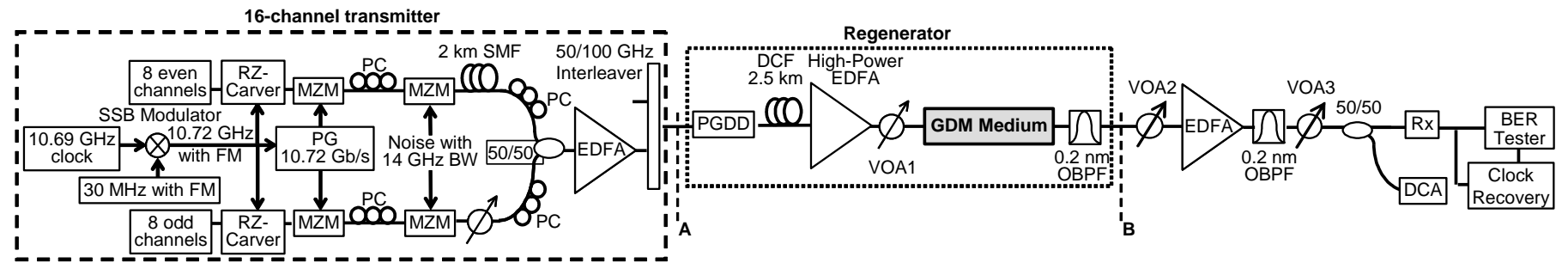

a

Group-delay-managed (GDM) nonlinear medium

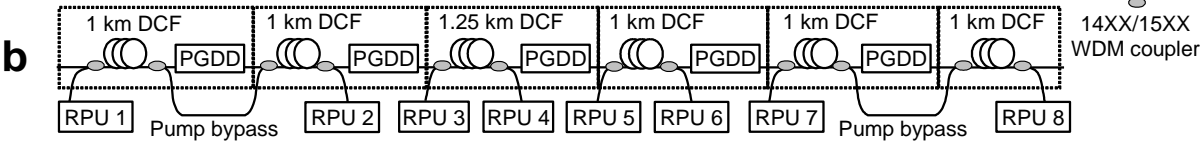

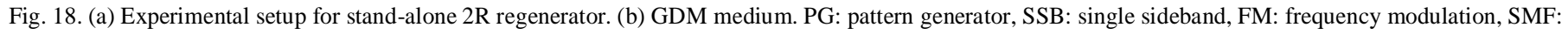
single-mode fiber, RPU: Raman pump unit.

The Mamyshev regenerator consists of the nonlinear GDM medium and an OBPF detuned by $-0.07 \mathrm{~nm}$ from the original channel's center. In a real system, a spectrally-periodic OBPF (comb filter such as an interleaver) can simultaneously handle all WDM channels, whereas we use a single tunable $0.2-\mathrm{nm}$ wide OBPF, as we only have one receiver. The GDM medium consists of 5 identical 1-km-long DCF sections and one 1.25$\mathrm{km}$ DCF section near the middle of the GDM, all separated by PGDDs. We use PGDDs based on cascaded Gires-Tournois etalons (PowerShaper ${ }^{\mathrm{TM}}$ PS3300 from II-VI, formerly Oclaro) [34], with $\approx 4 \mathrm{~dB}$ insertion loss each. The dispersion of each PGDD is set to $+150 \mathrm{ps} / \mathrm{nm}$, yielding the residual dispersion for each of 4 identical (1-km DCF + PGDD) unit cells near +30 $\mathrm{ps} / \mathrm{nm}$ optimum [42] and the residual dispersion of the cell with $1.25-\mathrm{km}$-long DCF near $0 \mathrm{ps} / \mathrm{nm}$. The net intra-channel dispersion of the entire GDM including the last piece of 1-kmlong DCF is $0 \mathrm{ps} / \mathrm{nm}$. At the same time, the signals from the adjacent $100-\mathrm{GHz}$-spaced WDM channels walk off from each other by at least 6 bit periods over the GDM length.

To compensate for the loss of the PGDDs, nonlinear fiber, couplers, and splices, totaling $\approx 35 \mathrm{~dB}$, and to achieve similar amounts of SPM in all fiber sections, we use bi-directional distributed Raman amplification (eight Raman pump units, or RPUs, each with two wavelengths and two polarizations multiplexed) in all sections of the nonlinear fiber. The optimum total average signal power entering the GDM medium is 26 $\mathrm{dBm}(\sim 25 \mathrm{~mW} / \mathrm{channel})$, and the total average signal power at the output of the last 1-km DCF section is $23.6 \mathrm{dBm}$. The multichannel regeneration is characterized by comparing the eye diagrams and "BER versus receiver input power" curves between the regenerator input (point A in Fig. 18a) and regenerator output (point B in Fig. 18a) for all channels.

The experimental results shown in Fig. 19 indicate similar amounts of the SPM-induced spectral broadening and similar eye-opening improvements $(4.8 \mathrm{~dB}$ or better) for all 16 regenerated channels [22]. Figure 20 shows a detailed view of one representative channel (channel 12) spectrum and its eye diagrams before and after regeneration.

The number of channels in this experiment is not constrained by any conceptual limitations of the GDM approach, and is only limited by the bandwidth of our Raman amplifiers, which depends on the pump laser wavelengths and powers available in our lab. Since an ideal PGDD is a pure allpass filter that can theoretically be $100 \%$ lossless, the use of lower-loss PGDDs and wider-bandwidth optical amplifiers can extend the GDM-based regeneration approach to higher channel counts typical for WDM communication systems. Other symbol rates are also possible after proper rescaling of the signal powers and GDM parameters [41], which has been numerically validated at $40 \mathrm{~Gb} / \mathrm{s}$ [41].

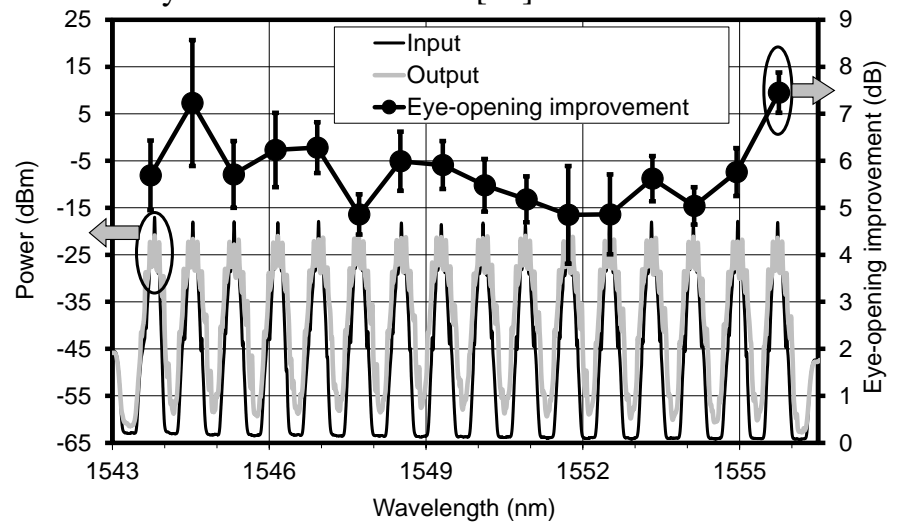

Fig. 19. (Left scale) Optical spectra of the noise-degraded signals at the standalone $2 \mathrm{R}$ regenerator input (thin black line) and output (thick gray line) for all 16 channels, obtained with resolution bandwidth of $0.016 \mathrm{~nm}$. (Right scale) Corresponding eye-opening improvements at BER $=10^{-9}$ level (filled black circles).

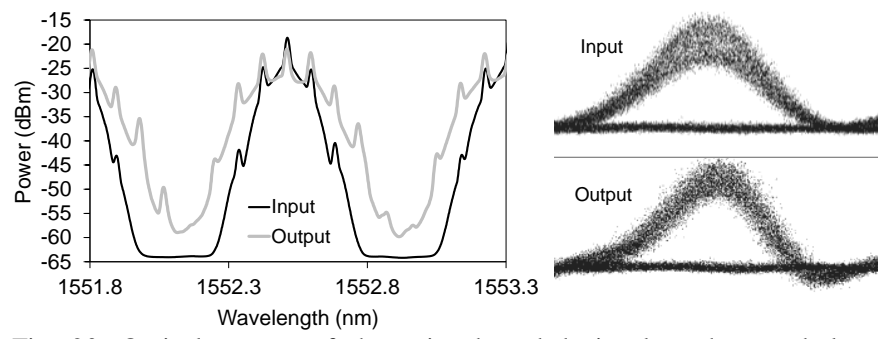

Fig. 20. Optical spectra of the noise-degraded signal at the stand-alone regenerator input (thin black line) and output (thick gray line) for one representative channel (channel 12 at $1552.51 \mathrm{~nm}$ ), obtained with resolution bandwidth of $0.016 \mathrm{~nm}$. Corresponding eye-diagrams are shown on the right.

Other SPM-based all-optical signal-processing devices, such as nonlinear optical loop mirrors (NOLM) [46, 47] can also utilize GDM medium, which opens the possibility for multi-channel phase-preserving amplitude regeneration of phase-encoded signals $[48,49]$ and multiple amplitude levels [50]. Such phase-preserving amplitude regenerators could further be paired $[51,52]$ with potentially multi-channel [5356] phase regenerators based on PSAs $[27,57,58]$ to provide multi-channel processing of signals encoded with advanced modulation formats, leading to the capacity and reach increase for communication systems [59]. 


\section{OUTLOOK FOR PRACTICAL DEPLOYMENT}

In addition to the WDM compatibility, the application of the all-optical regeneration in communication systems raises a number of other practical issues, most of which have been discussed in a detailed review [60]. For example, although the SPM-based regenerators are polarization-insensitive, their use with polarization-multiplexed signals may require polarization tracking. While in Sections III and IV we have discussed possible extensions of the two described multi-channel regeneration approaches to advanced modulation formats such as QPSK and quadrature amplitude modulation (QAM), both of these approaches are designed for RZ pulses, which are not typically used in modern coherent systems. The RZ pulses require the chromatic dispersion to be compensated prior to regeneration. In many modern transmission systems, optical dispersion compensation has been abandoned and replaced with digital dispersion compensation in coherent receivers. Hence, to be of practical use, the optical regenerators targeting these systems must provide a performance increase and cost savings that justify the added cost and complexity of optical dispersion compensation.

The WDM scalability of optical regenerators makes them attractive over optoelectronic regenerators in terms of cost, size, and power consumption. For example, with DCF as a nonlinear medium, adding one more regenerated WDM channel requires $\sim 25 \mathrm{~mW}$ of additional optical ( $50 \mathrm{~mW}$ electrical) power, whereas even the simplest (DSP-free) optoelectronic regenerator consumes at least $500 \mathrm{~mW}$ of electrical power just to drive Mach-Zehnder modulator.

While highly-nonlinear fiber provides today's best nonlinear figure of merit (nonlinearity / loss), several potential integrated nonlinear photonics platforms have emerged in the recent years. One of them is the ultra-silicon-rich nitride (USRN) [61] and the other is AlGaAs-on-insulator (AlGaAsOI) [37]. Both of these materials have been engineered to have nonlinearity similar to that of silicon, but with the bandgaps that are more than twice the energy of telecom-band photons. This avoids the detrimental two-photon absorption, which has severely limited the use of semiconductors such as silicon in optical signal processing, even with free-carrier sweeping employed [62]. Today's integrated devices already have a clear advantage over optical fiber in terms of processing bandwidth, but still trail in terms of optical losses and power handling ability. The high refractive indices of USRN and AlGaAsOI enable very small device size, while rapidly improving fabrication techniques will decrease propagation losses and enable integration of both nonlinear and linear components on the same photonic chip.

\section{REFERENCES}

[1] S. Watanabe, G. Ishikawa, T. Naito, and T. Chikama, "Generation of Optical Phase-Conjugate Waves and Compensation for Pulse Shape Distortion in a Single-Mode Fiber," J. Lightwave Technol., vol. 12, no. 12, pp. 2139-2146, 1994, doi: 10.1109/50.350616.

[2] X. Li et al., "Electronic post-compensation of WDM transmission impairments using coherent detection and digital signal processing," Opt. Express, vol. 16, no. 2, p. 880, Jan. 2008, doi: 10.1364/oe.16.000880.

[3] S. Rahbarfam and S. Kumar, "Nonlinear phase noise reduction using digital back propagation and midpoint optical phase conjugation," Opt. Express, vol. 27, no. 6, p. 8968, Mar. 2019, doi: 10.1364/oe.27.008968.
[4] F. Parmigiani et al., "Progress in multichannel all-optical regeneration based on fiber technology," IEEE J. Sel. Top. Quantum Electron., vol. 18, pp. 689-700, 2012.

[5] A. Shen et al., "4-channel Saturable Absorber Module for high bit-rate regenerated WDM transmission," in European Conf. Optical Communication, 2002, p. 5.4.5.

[6] Q. T. Le et al., "WDM compatible 2R regeneration device based on eightchannel saturable absorber module," Electron. Lett., vol. 43, no. 23, pp. 1305-1306, 2007, doi: 10.1049/el:20072745.

[7] F. Segnioean, B. Lavigne, D. Rouvillaio, P. Brindel, P. Pierre, and O. Leclerc, "Experimental demonstration of simple NOLM-based 2R regenerator for $42.66 \mathrm{Gbit} / \mathrm{s}$ WDM long-haul transmissions," in Optical Fiber Communication Conference, 2004.

[8] S. Boscolo, S. K. Turitsyn, and K. J. Blow, "All-optical passive 2R regeneration for $\mathrm{N} \times 40 \mathrm{Gbit} / \mathrm{s}$ WDM transmission using NOLM and novel filtering technique," Opt. Commun., vol. 217, no. 1-6, pp. 227-232, Mar. 2003, doi: 10.1016/S0030-4018(03)01123-4.

[9] K. Cvecek et al., "Phase-preserving amplitude regeneration for a WDM RZ-DPSK signal using a nonlinear amplifying loop mirror," Opt. Express, vol. 16, no. 3, p. 1923, Feb. 2008, doi: 10.1364/oe.16.001923.

[10] T. Ohara, H. Takara, A. Hirano, K. Mori, and S. Kawanishi, "40-Gb/s × 4-channel all-optical multichannel limiter utilizing spectrally filtered optical solitons," IEEE Photon. Technol. Lett., vol. 15, pp. 763-765, 2003.

[11] L. Provost, F. Parmigiani, P. Petropoulos, and D. J. Richardson, "Investigation of simultaneous $2 \mathrm{R}$ regeneration of two $40-\mathrm{Gb} / \mathrm{s}$ channels in a single optical fiber," IEEE Photon. Technol. Lett., vol. 20, pp. 270$272,2008$.

[12] P. Vorreau et al., "Optical grooming switch with regenerative functionality for transparent interconnection of networks," Opt. Express, vol. 17, pp. 15173-15185, 2009.

[13] L. Provost et al., "Investigation of four-wavelength regenerator using polarization- and direction-multiplexing," IEEE Photon. Technol. Lett., vol. 20, pp. 1676-1678, 2008.

[14] E. Desurvire, O. Leclerc, and O. Audouin, "Synchronous in-line regeneration of wavelength-division multiplexed solitons signals in optical fibers," Opt. Letters, vol. 21, no. 14, p. 1026, Jul. 1996, doi: 10.1364/ol.21.001026.

[15] N. Chi, L. Xu, K. S. Berg, T. Tokle, and P. Jeppesen, "All-optical wavelength conversion and multichannel $2 \mathrm{R}$ regeneration based on highly nonlinear dispersion-imbalanced loop mirror," IEEE Photon. Technol. Lett., vol. 14, no. 11, pp. 1581-1583, Nov. 2002, doi: 10.1109/LPT.2002.803343.

[16] N. S. M. Shah and M. Matsumoto, " 2 R Regeneration of time-interleaved multiwavelength signals based on higher order four-wave mixing in a fiber," IEEE Photon. Technol. Lett., vol. 22, no. 1, pp. 27-29, 2010, doi: 10.1109/LPT.2009.2035434

[17] Y.-K. Huang, I. Glesk, R. Shankar, and P. R. Prucnal, "Simultaneous alloptical 3R regeneration scheme with improved scalability using TOAD," Opt. Express, vol. 14, no. 22, p. 10339, Oct. 2006, doi: 10.1364/oe.14.010339.

[18] G. P. Agrawal, Nonlinear fiber optics. Elsevier, 2019.

[19] P. Guan et al., "Scalable WDM phase regeneration in a single phasesensitive amplifier through optical time lenses," Nature Commun., vol. 9, p. 1049, 2018, doi: 10.1038/s41467-018-03458-8.

[20] C. Kouloumentas et al., "Four-channel all-fibre dispersion-managed $2 \mathrm{R}$ regenerator," IEEE Photon. Technol. Lett., vol. 20, pp. 1169-1171, 2008.

[21] C. Kouloumentas et al., "All-fiberized dispersion-managed multichannel regeneration at $43 \mathrm{~Gb} / \mathrm{s}$," IEEE Photon. Technol. Lett., vol. 20, pp. 1854 1856,2008

[22] L. Li, P. G. Patki, Y. B. Kwon, V. Stelmakh, B. D. Campbell, M. Annamalai, T. I. Lakoba, and M. Vasilyev, "All-optical regenerator of multi-channel signals," Nature Commun., vol. 8, p. 884, 2017.

[23] B. H. Kolner and M. Nazarathy, "Temporal imaging with a time lens," Opt. Lett., vol. 14, no. 12, p. 630, Jun. 1989, doi: 10.1364/ol.14.000630.

[24] M. Nakazawa, T. Hirooka, F. Futami, and S. Watanabe, "Ideal distortionfree transmission using optical Fourier transformation and Fourier transform-limited optical pulses," IEEE Photon. Technol. Lett., vol. 16, no. 4, pp. 1059-1061, Apr. 2004, doi: 10.1109/LPT.2004.824969.

[25] E. Palushani et al., "OTDM-to-WDM conversion based on time-tofrequency mapping by time-domain optical fourier transformation," IEEE J. Sel. Top. Quant. Electron., vol. 18, no. 2, pp. 681-688, 2012, doi: 10.1109/JSTQE.2011.2131121.

[26] H. Christian Hansen et al., "DWDM-TO-OTDM Conversion by TimeDomain Optical Fourier Transformation," Optical Society of America, 
2011. Accessed: Jun. 11, 2020. [Online]. Available: http://www.ecoc2011.org/.

[27] R. Slavík et al., "All-optical phase and amplitude regenerator for nextgeneration telecommunications systems," Nature Photon., vol. 4, no. 10, pp. 690-695, Oct. 2010, doi: 10.1038/nphoton.2010.203.

[28] C. Lundström, Z. Tong, M. Karlsson, and P. A. Andrekson, "Phase-tophase and phase-to-amplitude transfer characteristics of a nondegenerateidler phase-sensitive amplifier," Opt. Lett., vol. 36, no. 22, p. 4356, Nov. 2011, doi: 10.1364/ol.36.004356.

[29] N. K. Kjoller, K. M. Roge, P. Guan, H. C. H. Mulvad, M. Galili, and L. K. Oxenlowe, "A Novel Phase-Locking-Free Phase Sensitive AmplifierBased Regenerator," J. Lightwave Technol., vol. 34, no. 2, pp. 643-652, Jan. 2016, doi: 10.1109/JLT.2015.2482122.

[30] N. K. Kjøller, F. da Ros, K. M. Røge, P. Guan, M. Galili, and L. K. Oxenløwe, "A novel phase sensitive amplifier based QPSK regenerator without active phase-locking," in 2015 IEEE Photonics Conference, IPC 2015, Nov. 2015, pp. 252-253, doi: 10.1109/IPCon.2015.7323558.

[31] P. V. Mamyshev, "All-optical data regeneration based on self-phase modulation effect," Proc. of the European Conference on Optical Communications, Madrid, Spain, 1998, pp. 475-476.

[32] R. L. Lachance, S. Lelievre, and Y. Painchaud, "50 and $100 \mathrm{GHz}$ multichannel tunable chromatic dispersion slope compensator," Proc. of the Optical Fiber Communications Conference 2003, Technical Digest (OSA, Washington, D.C. 2003), Vol. 1, pp. 164-165.

[33] L. M. Lunardi et al., "Tunable dispersion compensation at $40 \mathrm{~Gb} / \mathrm{s}$ using a multicavity etalon all-pass filter, with NRZ, RZ and CSRZ modulation," J. Lightwave Technol., vol. 20, pp. 2136-2144, 2002.

[34] D. Yang, C. Lin, W. Chen, and G. Barbarossa, "Fiber dispersion and dispersion slope compensation in a 40-channel $10-\mathrm{Gb} / \mathrm{s} 3200-\mathrm{km}$ transmission experiment using cascaded single-cavity Gires-Tournois etalons," IEEE Photon. Technol. Lett., vol. 16, pp. 299-301, 2004.

[35] B. J. Eggleton, B. Luther-Davies, and K. Richardson, "Chalcogenide photonics," Nature Photon., vol. 5, pp. 141-148, 2011.

[36] D. J. Moss, R. Morandotti, A. L. Gaeta, and M. Lipson, "New CMOScompatible platforms based on silicon nitride and Hydex for nonlinear optics," Nature Photon., vol. 7, pp. 597-607, 2013.

[37] M. Pu et al., "Ultra-Efficient and Broadband Nonlinear AlGaAs-onInsulator Chip for Low-Power Optical Signal Processing," Laser and Photon. Rev., vol. 12, no. 12, p. 1800111, Dec. 2018, doi: 10.1002/lpor.201800111

[38] C. K. Madsen et al., "Integrated all-pass filters for tunable dispersion and dispersion slope compensation," IEEE Photon. Technol. Lett., vol. 11, pp. $1623-1625,1999$.

[39] C. R. Doerr, L. W. Stulz, S. Chandrasekhar, L. Buhl, and R. Pafchek, "Multichannel integrated tunable dispersion compensator employing a thermooptic lens," Proc. of the Optical Fiber Communications Conference, Anaheim, CA, March 17-22, 2002, paper PD FA6.

[40] N. Stelmakh and M. Vasilyev, "Multiport AWG-based dispersion compensators," Proc. of the IEEE Photonics Society Summer Topical Meeting on Novel Waveguiding, Structures, and Phenomena, Playa del Carmen, Mexico, July 19-21, 2010, paper WB2.5.

[41] M. Vasilyev and T. I. Lakoba, "All-optical multichannel $2 \mathrm{R}$ regeneration in a fiber-based device," Opt. Lett., vol. 30, pp. 1458-1460, 2005.

[42] T. I. Lakoba and M. Vasilyev, "A new robust regime for a dispersionmanaged multichannel 2R regenerator," Opt. Express, vol. 15, pp. 1006110074, 2007

[43] P. G. Patki, M. Vasilyev, and T. I. Lakoba, "All-optical regeneration of multi-wavelength signals," Proc. of the IEEE LEOS European Winter Topical on Nonlinear Processing in Optical Fibres, Innsbruck, Austria, January 12-14, 2009, paper WC2.3.

[44] P. G. Patki, M. Vasilyev, and T. I. Lakoba, "Multichannel all-optical regeneration," Proc. of the IEEE Photonics Society Summer Topical Meeting on Nonlinear Fiber Optics, Playa del Carmen, Mexico, July 1921, 2010, paper WC2.2.

[45] L. Li, Y.-B. Kwon, B. Campbell, T. I. Lakoba, and M. Vasilyev, "2R regeneration of 12 WDM channels with $100-\mathrm{GHz}$ spacing in a groupdelay-managed nonlinear medium," Proc. of the Optical Fiber Communication conference, Annaheim, CA, March 20-24, 2016, paper W4D.4.

[46] S. Boscolo, R. Bhamber, and S. K. Turitsyn, "Design of Raman-based nonlinear loop mirror for all-optical $2 \mathrm{R}$ regeneration of differential phaseshift-keying transmission," IEEE J. Quantum Electron., vol. 42, pp. 619624, 2006.

[47] K. Cvecek, K. Sponsel, G. Onishchukov, B. Schmauss, and G. Leuchs, "2R-Regeneration of an RZ-DPSK signal using a nonlinear amplifying loop mirror," IEEE Photon. Technol. Lett., vol. 19, pp. 146-148, 2007.

[48] T. I. Lakoba, J. R. Williams, and M. Vasilyev, "NALM-based, phasepreserving $2 \mathrm{R}$ regenerator of high-duty-cycle pulses," Opt. Express, vol. 19, pp. 23017-23028, 2011

[49] T. I. Lakoba and M. Vasilyev, "On multi-channel operation of phasepreserving 2R amplitude regenerator," Opt. Commun., vol. 322, pp. 114$117,2014$.

[50] T. Röthlingshöfer et al., "All-optical phase-preserving multilevel amplitude regeneration," Opt. Express, vol. 22, pp. 27077-27085, 2014.

[51] L. Li and M. Vasilyev, "All-optical 2R regenerator of 16-QAM signals," Proc. SPIE, vol. 9009, p. 900908, 2014.

[52] M. Sorokina, S. Sygletos, A. Ellis, and S. Turitsyn, "Regenerative Fourier transformation for dual-quadrature regeneration of multilevel rectangular QAM,” Opt. Lett., vol. 40, pp. 3117-3120, 2015.

[53] M. Vasilyev, "Distributed phase-sensitive amplification," Opt. Express, vol. 13, pp. 7563-7571, 2005.

[54] R. Tang, P. S. Devgan, V. S. Grigoryan, P. Kumar, and M. Vasilyev, "Inline phase-sensitive amplification of multi-channel $\mathrm{CW}$ signals based on frequency non-degenerate four-wave-mixing in fiber," Opt. Express, vol. 16, pp. 9046-9053, 2008.

[55] S. Sygletos et al., "A practical phase sensitive amplification scheme for two channel phase regeneration," Opt. Express, vol. 19, pp. B938-B945, 2011

[56] F. Parmigiani, K. R. H. Bottrill, R. Slavík, D. J. Richardson, and P. Petropoulos, "PSA-based all-optical multi-channel phase regenerator," Proc. of the European Conference on Optical Communications, Valencia, Spain, September 27 - October 1, 2015, paper P.3.13.

[57] Z. Tong et al., "Towards ultrasensitive optical links enabled by low-noise phase-sensitive amplifiers," Nature Photon., vol. 5, pp. 430-436, 2011.

[58] J. Kakande et al., "Multilevel quantization of optical phase in a novel coherent parametric mixer architecture," Nature Photon., vol. 5, pp. 748$752,2011$.

[59] M. A. Sorokina and S. K. Turitsyn, "Regeneration limit of classical Shannon capacity," Nature Commun., vol. 5, p. 3861, 2014.

[60] E. Ciaramella, "Wavelength conversion and all-optical regeneration: achievements and open issues," J. Lightwave Technol., vol. 30, pp. 572$582,2012$.

[61] K. J. A. Ooi et al, "Pushing the limits of CMOS optical parametric amplifiers with USRN: $\mathrm{Si}_{7} \mathrm{~N}_{3}$ above the two-photon absorption edge," Nature Commun., vol. 7, p. 13878, 2017.

[62] F. da Ros et al., "Dual-polarization wavelength conversion of 16-QAM signals in a single silicon waveguide with a lateral p-i-n diode [Invited]," Photon. Research, vol. 6, no. 5, p. B23, May 2018, doi: 10.1364/prj.6.000b23.

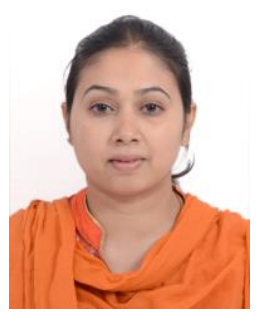

Pallavi G. Patki received her M.S. in 2006 and Ph.D. in 2010 in electrical engineering from the University of Texas at Arlington, where she studied multi-channel all-optical regeneration in a recirculating loop.

After spending several years as an optical systems engineer at Xtera Communications in Allen, TX, where she worked on unrepeatered and ultra-long-haul WDM transmission systems, she joined Infinera in Bangalore, India in 2015, where she is currently a staff systems engineer.

Dr. Patki's interests include WDM optical communication systems, optical interconnects, optical signal processing, and nonlinear optics.

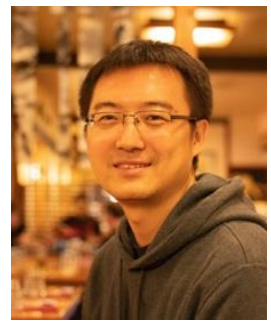

Pengyu Guan received M.S. degree and Ph.D. degree in communications engineering from Tohoku University, Sendai, Japan, in 2009 and 2012. Currently he is a Senior researcher in the High-Speed Optical Communications Group, DTU Fotonik, where he is involved in research on high-speed optical signal processing and terabit/s optical communication systems. He has authored 
or co-authored 76 peer reviewed publications, and holds 4 patents as the first inventor. He is the recipient of a very prestigious young investigator grant from VILLUM FONDEN for the project LENS-PON. His research interests include novel optical communications system, nonlinear optical signal processing and time lens based optical Fourier transformation. Dr. Guan is a member of both OSA and IEEE.

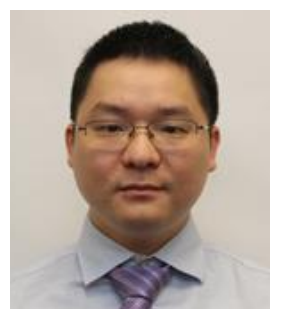

Lu Li received his B.S. in optoelectronics from Nankai University in 2007, M.S. in optoelectronics from Tianjin University in 2010, and Ph.D. in electrical engineering from the University of Texas at Arlington in 2016. In his Ph.D. work, he investigated multi-channel optical regeneration.

Since joining SubCom in Eatontown, New Jersey in 2017 as a Senior Member of Technical Staff, he has worked on transoceanic cable system design and optimization. His current interests include all-optical processing, nonlinear propagation, as well as optical amplifiers and ROADMs in undersea transmission systems.

$\mathrm{Dr}$. $\mathrm{Li}$ is a member of the technical program committee for Optical Fiber Communication conference.

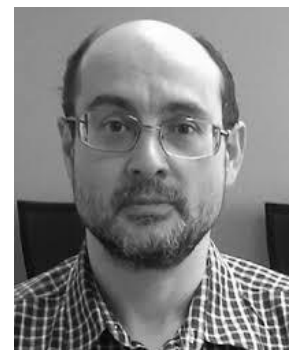

Taras Lakoba received his M.S. degree in physics from Moscow State University in 1989 and Ph.D. degree in applied mathematics from Clarkson University in 1996.

He worked as a postdoctoral researcher at the University of Rochester and University of Central Florida, as well as a Member of Technical Staff at Bell Labs, Lucent Technologies, where he analyzed and simulated LambdaXtreme, an ultra-long-haul WDM transmission system. Since 2003 he is with the Department of Mathematics and Statistics, University of Vermont, Burlington, VT, USA, where he is currently a Professor,

Prof. Lakoba's research interests include mathematical modeling of optical propagation in telecommunication fibers and photonic devices and development of numerical methods for nonlinear wave equations.

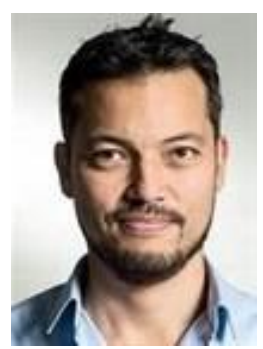

Leif Katsuo Oxenløwe received the B.Sc. degree in physics and astronomy from the Niels Bohr Institute, University of Copenhagen, Denmark in 1996. In 1998 he received the International Diploma of Imperial College, London, UK and the M.Sc. degree from the University of Copenhagen. He received the Ph.D. degree in 2002 from the Technical University of Denmark (DTU) and is since 2009 Professor of Photonic Communication Technologies. He is the group leader of the High-Speed Optical Communications group at DTU Fotonik, Department of Photonics Engineering, at DTU, and the Centre Leader of the Research Centre of Excellence SPOC (Silicon Photonics for Optical Communications) supported by the Danish National Research Foundation. He is the recipient of a
Top-Researcher grant from the Danish Research Council (DFF) for the project NANO-SPECs. He is also the recipient of a European Research Council grant (project SOCRATES) focusing on the use of ultra-high-speed serial data for Ethernet networks, and he is involved in several other national and international projects exploring nonlinear optics in fibres and devices for optical signal processing. He has authored or coauthored more than 350 peer reviewed publications, including 15 postdeadline papers at major conferences and 5 book chapters. He holds 7 patents, and he has received 4 best paper awards. In 2016, SPOC won The Horizon Prize - Breaking the optical transmission barriers with the proposal PHOTONMAP (PHOtonic chip enabled large-capacity data TransmissiON with high- count Multi-core fibres and AmPlifiers) https://ec.europa.eu/research/horizonprize/index.cfm?prize=op ticaltransmission

Prof. Oxenløwe is a fellow of the OSA and he is the 2009 recipient of the Elektropris from Elektrofondet.

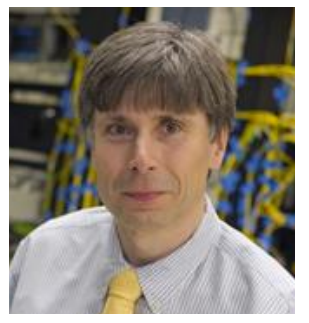

Michael Vasilyev received M.S. in physics from Moscow Institute of Physics and Technology in 1993 and Ph.D. in electrical engineering from Northwestern University in 1999.

Prior to joining the Department of Electrical Engineering at the University of Texas at Arlington in 2003, where he is currently a Professor, he was a senior research scientist at Corning, Inc., investigating noise and nonlinearities in EDFAs and Raman amplifiers to optimize ultra-long-haul transmission systems. His research interests include novel optical amplifiers, nonlinear-optical signal processing, quantum communications, and nanophotonics.

Prof. Vasilyev is a Fellow of the OSA and SPIE, is a recipient of 2008 DARPA Young Faculty Award, has over 200 publications, and holds 10 patents.

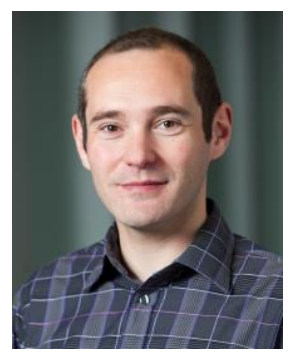

Michael Galili obtained his M.S. in Engineering in applied physics, from the Technical University of Denmark (DTU), in 2003 and his Ph.D. in Optical communications and signal processing from DTU Fotonik in 2007 where he is currently Associate Professor. He also servers as scientific coordinator in the research center Silicon Photonics for Optical Communication (SPOC). He is the first author or coauthor of more than 300 peer reviewed journal and conference publications. His research interests include optical transmission and signal processing for telecommunication, nonlinear integrated devices for optical processing and advanced optical switches for datacom systems.

Dr. Galili is a member of both the OSA and the IEEE and recipient of the Gorm Petersen award in 2007. 